\title{
Global Stability of a Discrete Mutualism Model
}

\author{
Kun Yang, ${ }^{1}$ Xiangdong Xie, ${ }^{2}$ and Fengde Chen ${ }^{1}$ \\ ${ }^{1}$ College of Mathematics and Computer Science, Fuzhou University, Fuzhou, Fujian 350116, China \\ ${ }^{2}$ Department of Mathematics, Ningde Normal University, Fujian 352100, China
}

Correspondence should be addressed to Xiangdong Xie; ndsyxxd@163.com

Received 24 April 2014; Accepted 10 June 2014; Published 23 June 2014

Academic Editor: Luca Guerrini

Copyright (C) 2014 Kun Yang et al. This is an open access article distributed under the Creative Commons Attribution License, which permits unrestricted use, distribution, and reproduction in any medium, provided the original work is properly cited.

\begin{abstract}
A discrete mutualism model is studied in this paper. By using the linear approximation method, the local stability of the interior equilibrium of the system is investigated. By using the iterative method and the comparison principle of difference equations, sufficient conditions which ensure the global asymptotical stability of the interior equilibrium of the system are obtained. The conditions which ensure the local stability of the positive equilibrium is enough to ensure the global attractivity are proved.
\end{abstract}

\section{Introduction}

There are many examples where the interaction of two or more species is to the advantage of all; we call such a situation the mutualism. For example, cellulose of white ants' gut provides nutrients for flagellates, while flagellates provide nutrients for white ants through the decomposition of cellulose to glucose. As was pointed out by Chen et al. [1] "the mutual advantage of mutualism or symbiosis can be very important. As a topic of theoretical ecology, even for two species, this area has not been as widely studied as the others even though its importance is comparable to that of predatorprey and competition interactions." Thus, it seems interesting to study some relevant topics on the symbiosis system.

The following model was proposed by Chen et al. [1] to describe the mutualism mechanism:

$$
\begin{aligned}
& \frac{d N_{1}}{d t}=r_{1} N_{1}(t)\left[\frac{K_{1}+\alpha_{1} N_{2}(t)}{1+N_{2}(t)}-N_{1}(t)\right], \\
& \frac{d N_{2}}{d t}=r_{2} N_{2}(t)\left[\frac{K_{2}+\alpha_{2} N_{1}(t)}{1+N_{1}(t)}-N_{2}(t)\right],
\end{aligned}
$$

where $r_{i}$ refers to the intrinsic rate of population $N_{i}$ and $\alpha_{i}>$ $K_{i}(i=1,2)$. In the absence of other species, the carrying capacity of the species $N_{i}$ is $K_{i}$. Thanks to the cooperation of the other species, the carrying capacity of the species $N_{i}$ becomes $\left(K_{i}+\alpha_{i} N_{3-i}\right) /\left(1+N_{3-i}\right)$.
$\mathrm{Li}$ [2] argued that the nonautonomous one is more appropriate, and he proposed the following two-species cooperative model:

$$
\begin{aligned}
\frac{d N_{1}}{d t}= & r_{1}(t) N_{1}(t) \\
& \times\left[\frac{K_{1}(t)+\alpha_{1}(t) N_{2}\left(t-\tau_{2}(t)\right)}{1+N_{2}\left(t-\tau_{2}(t)\right)}-N_{1}\left(t-\sigma_{1}(t)\right)\right], \\
\frac{d N_{2}}{d t}= & r_{2}(t) N_{2}(t) \\
& \times\left[\frac{K_{2}(t)+\alpha_{2}(t) N_{1}\left(t-\tau_{1}(t)\right)}{1+N_{1}\left(t-\tau_{1}(t)\right)}-N_{2}\left(t-\sigma_{2}(t)\right)\right],
\end{aligned}
$$

where $r_{i}, K_{i}, \alpha_{i}, \tau_{i}, \sigma_{i} \in C(R, R+), \alpha_{i}>K_{i}, r_{i}, K_{i}, \alpha_{i}, \tau_{i}, \sigma_{i}(i=$ $1,2)$ are periodic functions of period $\omega>0$. Here the author incorporates the time delays to the model, which means that the cooperation effect needs to spend some time to realize, but not immediately realize. By applying the coincidence degree theory, Li showed that the system has at least one positive periodic solution. For more works related to the system (1) and (2), one could refer to [1-9] and the references cited therein.

It is well known that the discrete time models governed by difference equations are more appropriate than the continuous ones when the populations have nonoverlapping 
generations, and discrete time models can also provide efficient computational models of continuous models for numerical simulations. Corresponding to system (2), Li [10] proposed the following delayed discrete model of mutualism:

$$
\begin{aligned}
& x_{1}(k+1) \\
& =x_{1}(k) \exp \left\{r _ { 1 } ( k ) \left[\frac{K_{1}(k)+\alpha_{1}(k) x_{2}\left(k-\tau_{2}(k)\right)}{1+x_{2}\left(k-\tau_{2}(k)\right)}\right.\right. \\
& \left.\left.-x_{1}\left(k-\sigma_{1}(k)\right)\right]\right\}, \\
& x_{1}(k+1) \\
& =x_{1}(k) \exp \left\{r _ { 1 } ( k ) \left[\frac{K_{2}(k)+\alpha_{2}(k) x_{1}\left(k-\tau_{1}(k)\right)}{1+x_{1}\left(k-\tau_{1}(k)\right)}\right.\right. \\
& \left.\left.-x_{2}\left(k-\sigma_{2}(k)\right)\right]\right\} .
\end{aligned}
$$

Under the assumption that $r_{i}, K_{i}, \alpha_{i}, \tau_{i}, \sigma_{i}(i=1,2)$ are positive periodic sequences with a common cycle $\omega$, and $\alpha_{i}>K_{i}$ holds, by applying coincidence degree theory, he showed that system (3) has at least one positive $\omega$-periodic solution, where $\omega$ is a positive integer. Chen [11] argued that the general nonautonomous nonperiodic system is more appropriate, and he showed that the system (3) is permanent. For more work about cooperative system, we can refer to [1219].

It brings to our attention that neither $\mathrm{Li}$ [10] nor Chen [11] investigated the stability property of the system (3), which is one of the most important topics on the study of population dynamics. We mention here that, with $\sigma_{i}(k) \neq 0$, system $(3)$ is a pure-delay system, and it is not an easy thing to investigate the stability property of the system. This motivated us to discuss a simple system, that is, the following autonomous cooperative system:

$$
\begin{aligned}
& x_{1}(k+1)=x_{1}(k) \exp \left\{r_{1}\left[\frac{K_{1}+\alpha_{1} x_{2}(k)}{1+x_{2}(k)}-x_{1}(k)\right]\right\}, \\
& x_{2}(k+1)=x_{2}(k) \exp \left\{r_{2}\left[\frac{K_{2}+\alpha_{2} x_{1}(k)}{1+x_{1}(k)}-x_{2}(k)\right]\right\},
\end{aligned}
$$

where $x_{i}(k)(i=1,2)$ are the population density of the $i$ th species at $k$-generation. $r_{i}, K_{i}, i=1,2$, are all positive constants.

Throughout this paper, we assume that the coefficients of system (4) satisfy

$$
\left(H_{1}\right) r_{i}, K_{i}, \alpha_{i}(i=1,2) \text { are all positive constants and } \alpha_{i}>
$$
$K_{i}(i=1,2)$.

The aim of this paper is, by further developing the analysis technique of [15], to obtain a set of sufficient conditions to ensure the global asymptotical stability of the interior equilibrium of system (4). More precisely, we will prove the following result.

Theorem 1. In addition to $\left(H_{1}\right)$, further assume that

$$
\left(H_{2}\right) r_{i} \alpha_{i} \leq 1,(i=1,2)
$$

holds; then the unique positive equilibrium $\left(x_{1}^{*}, x_{2}^{*}\right)$ of the system (4) is globally asymptotically stable.

The rest of the paper is arranged as follows. In Section 2 we will introduce a useful lemma and investigate the local stability property of the positive equilibrium. With the help of several useful lemmas, the global attractivity of positive equilibrium of the system (4) is investigated in Section 3. An example together with its numeric simulation is presented in Section 4 to show the feasibility of our results. We end this paper by a brief discussion.

\section{Local Stability}

In view of the actual ecological implications of system (4), we assume that the initial value $x_{i}(0)>0(i=1,2)$. Obviously, any solution of system (4) with positive initial condition is well defined on $Z_{+}$, where $Z_{+}=\{0,1,2, \ldots\}$ and remains positive for all $n \geq 0$.

We determine the positive equilibrium of the system (4) through solving the following equations:

$$
\begin{aligned}
& x_{1}=x_{1} \exp \left[r_{1}\left(\frac{K_{1}+\alpha_{1} x_{2}}{1+x_{2}}-x_{1}\right)\right], \\
& x_{2}=x_{2} \exp \left[r_{2}\left(\frac{K_{2}+\alpha_{2} x_{1}}{1+x_{1}}-x_{2}\right)\right],
\end{aligned}
$$

which is equivalent to

$$
x_{1}=\frac{K_{1}+\alpha_{1} x_{2}}{1+x_{2}}, \quad x_{2}=\frac{K_{2}+\alpha_{2} x_{1}}{1+x_{1}},
$$

and so

$$
\begin{gathered}
\left(\alpha_{1}+1\right) x_{2}^{2}+\left(1-\alpha_{1} \alpha_{2}+K_{1}-K_{2}\right) x_{2} \\
-\left(K_{2}+\alpha_{2} K_{1}\right)=0 .
\end{gathered}
$$

Since $\alpha_{1}+1>0, K_{2}+\alpha_{2} K_{1}>0$, (7) admits a unique positive solution $\bar{x}_{2}$. From the first equation of system (6), one could obtain $\bar{x}_{1}$; therefore, system (4) admits a unique positive equilibrium $E_{+}\left(\bar{x}_{1}, \bar{x}_{2}\right)$.

Following we will discuss the local stability of equilibrium $E_{+}\left(\bar{x}_{1}, \bar{x}_{2}\right)$. The Jacobian matrix of system (4) at $E_{+}\left(\bar{x}_{1}, \bar{x}_{2}\right)$ is as follows:

$$
J\left(E_{+}\right)=\left(\begin{array}{cc}
1-r_{1} \bar{x}_{1} & r_{2} \bar{x}_{2} \frac{\alpha_{2}-K_{2}}{\left(1+\bar{x}_{1}\right)^{2}} \\
r_{1} \bar{x}_{1} \frac{\alpha_{1}-K_{1}}{\left(1+\bar{x}_{2}\right)^{2}} & 1-r_{2} \bar{x}_{2}
\end{array}\right) .
$$

The characteristic equation of $J\left(E_{+}\right)$is

$$
F(\lambda)=\lambda^{2}+B \lambda+C=0
$$

where

$$
\begin{aligned}
B= & -\left(2-r_{1} \bar{x}_{1}-r_{2} \bar{x}_{2}\right), \\
C= & \left(1-r_{1} \bar{x}_{1}\right)\left(1-r_{2} \bar{x}_{2}\right) \\
& -r_{1} r_{2} \bar{x}_{1} \bar{x}_{2} \frac{\left(\alpha_{1}-K_{1}\right)\left(\alpha_{2}-K_{2}\right)}{\left(1+\bar{x}_{1}\right)^{2}\left(1+\bar{x}_{2}\right)^{2}} .
\end{aligned}
$$


Lemma 2 (see [20]). Let $F(\lambda)=\lambda^{2}+B \lambda+C=0$, where $B$ and $C$ are constants. Suppose $F(1)>0$ and $\lambda_{1}, \lambda_{2}$ are two roots of $F(\lambda)=0$. Then $\left|\lambda_{1}\right|<1$ and $\left|\lambda_{2}\right|<1$ if and only if $F(-1)>0$ and $C<1$.

Let $\lambda_{1}$ and $\lambda_{2}$ be the two roots of (9), which are called eigenvalues of equilibrium $E_{+}\left(\bar{x}_{1}, \bar{x}_{2}\right)$. From [20] we know that if $\left|\lambda_{1}\right|<1$ and $\left|\lambda_{2}\right|<1$, then $E_{+}\left(\bar{x}_{1}, \bar{x}_{2}\right)$ is locally asymptotically stable.

Theorem 3. Assume that $\left(H_{1}\right)$ and $\left(H_{2}\right)$ hold; then $E_{+}\left(\bar{x}_{1}, \bar{x}_{2}\right)$ is locally asymptotically stable.

Proof. Since (7) has two real solutions, it follows that its discriminant is positive; that is,

$$
\begin{aligned}
\Delta & =\left(1-\alpha_{1} \alpha_{2}+K_{1}-K_{2}\right)^{2}+4\left(\alpha_{1}+1\right)\left(K_{2}+\alpha_{2} K_{1}\right) \\
& =\left(1+\alpha_{1} \alpha_{2}+K_{1}+K_{2}\right)^{2}-4\left(\alpha_{1}-K_{1}\right)\left(\alpha_{2}-K_{2}\right)>0
\end{aligned}
$$

and so

$$
\left(1+\alpha_{1} \alpha_{2}+K_{1}+K_{2}\right)^{2}>4\left(\alpha_{1}-K_{1}\right)\left(\alpha_{2}-K_{2}\right) .
$$

Equation (6) combined with the above inequality implies that

$$
\begin{aligned}
\left(1+\bar{x}_{1}\right)^{2}\left(1+\bar{x}_{2}\right)^{2} \\
=\left(1+\frac{K_{1}+\alpha_{1} \bar{x}_{2}}{1+\bar{x}_{2}}\right)^{2}\left(1+\bar{x}_{2}\right)^{2} \\
=\left(\left(\alpha_{1}+1\right) \bar{x}_{2}+\left(K_{1}+1\right)\right)^{2} \\
=\left(\frac{1+\alpha_{1} \alpha_{2}+K_{1}+K_{2}+\sqrt{\Delta}}{2}\right)^{2} \\
>\frac{\left(1+\alpha_{1} \alpha_{2}+K_{1}+K_{2}\right)^{2}}{4}=\left(\alpha_{1}-K_{1}\right)\left(\alpha_{2}-K_{2}\right) . \\
>\frac{4\left(\alpha_{1}-K_{1}\right)\left(\alpha_{2}-K_{2}\right)}{4}
\end{aligned}
$$

The above inequality leads to

$$
1-\frac{\left(\alpha_{1}-K_{1}\right)\left(\alpha_{2}-K_{2}\right)}{\left(1+\bar{x}_{1}\right)^{2}\left(1+\bar{x}_{2}\right)^{2}}>0 .
$$

From (9) and (14) it follows that

$$
\begin{aligned}
F(1) & =1+B+C \\
& =r_{1} r_{2} \bar{x}_{1} \bar{x}_{2}\left(1-\frac{\left(\alpha_{1}-K_{1}\right)\left(\alpha_{2}-K_{2}\right)}{\left(1+\bar{x}_{1}\right)^{2}\left(1+\bar{x}_{2}\right)^{2}}\right)>0 .
\end{aligned}
$$

Also, from (6) and $\left(H_{2}\right)$, one has

$$
\bar{x}_{i}<\alpha_{i}, \quad r_{i} \bar{x}_{i}<1 \quad(i=1,2) .
$$

According to inequality (14) and (16), we have

$$
\begin{aligned}
F(-1)= & 1-B+C \\
= & r_{1} r_{2} \bar{x}_{1} \bar{x}_{2}\left(1-\frac{\left(\alpha_{1}-K_{1}\right)\left(\alpha_{2}-K_{2}\right)}{\left(1+\bar{x}_{1}\right)^{2}\left(1+\bar{x}_{2}\right)^{2}}\right) \\
& +\left(4-2 r_{1} \bar{x}_{1}-2 r_{2} \bar{x}_{2}\right)>0, \\
C-1= & -r_{1} \bar{x}_{1}-r_{2} \bar{x}_{2} \\
& +r_{1} r_{2} \bar{x}_{1} \bar{x}_{2}\left(1-\frac{\left(\alpha_{1}-K_{1}\right)\left(\alpha_{2}-K_{2}\right)}{\left(1+\bar{x}_{1}\right)^{2}\left(1+\bar{x}_{2}\right)^{2}}\right) \\
< & -r_{1} \bar{x}_{1}\left(1-r_{2} \bar{x}_{2}\right)-r_{2} \bar{x}_{2}<0 .
\end{aligned}
$$

From Lemma 2 we can obtain that $E_{+}\left(\bar{x}_{1}, \bar{x}_{2}\right)$ is locally asymptotically stable. This completes the proof of Theorem 3 .

\section{Global Stability}

We will give a strict proof of Theorem 1 in this section. To achieve this objective, we introduce several useful lemmas.

Lemma 4 (see [20]). Let $f(u)=u \exp (\alpha-\beta u)$, where $\alpha$ and $\beta$ are positive constants; then $f(u)$ is nondecreasing for $u \in$ $(0,(1 / \beta)]$.

Lemma 5 (see [20]). Assume that sequence $\{u(k)\}$ satisfies

$$
u(k+1)=u(k) \exp (\alpha-\beta u(k)), \quad k=1,2, \ldots,
$$

where $\alpha$ and $\beta$ are positive constants and $u(0)>0$. Then

(i) if $\alpha<2$, then $\lim _{k \rightarrow+\infty} u(k)=\alpha / \beta$;

(ii) if $\alpha<1$, then $u(k) \leq(1 / \beta), k=2,3, \ldots$.

Lemma 6 (see [21]). Suppose that functions $f, g: Z_{+} \times$ $[0, \infty) \rightarrow[0, \infty)$ satisfy $f(k, x) \leq g(k, x)(f(k, x) \geq g(k, x))$ for $k \in Z_{+}$and $x \in[0, \infty)$ and $g(k, x)$ is nondecreasing with respect to $x$. If $\{x(k)\}$ and $\{u(k)\}$ are the nonnegative solutions of the difference equations

$$
x(k+1)=f(k, x(k)), \quad u(k+1)=g(k, u(k)),
$$

respectively, and $x(0) \leq u(0)(x(0) \geq u(0))$, then

$$
x(k) \leq u(k)(x(k) \geq u(k)) \quad \forall k \geq 0 .
$$

Proof of Theorem 1. Let $\left(x_{1}(k), x_{2}(k)\right)$ be arbitrary solution of system (4) with $x_{1}(0)>0$ and $x_{2}(0)>0$. Denote

$$
\begin{array}{ll}
U_{1}=\limsup _{k \rightarrow+\infty} x_{1}(k), & V_{1}=\liminf _{k \rightarrow+\infty} x_{1}(k), \\
U_{2}=\limsup _{k \rightarrow+\infty} x_{2}(k), & V_{2}=\liminf _{k \rightarrow+\infty} x_{2}(k) .
\end{array}
$$

We claim that $U_{1}=V_{1}=\bar{x}_{1}$ and $U_{2}=V_{2}=\bar{x}_{2}$. 
From the first equation of system (4), we obtain

$$
\begin{array}{r}
x_{1}(k+1) \leq x_{1}(k) \exp \left\{r_{1} \alpha_{1}-r_{1} x_{1}(k)\right\}, \\
k=0,1,2, \ldots
\end{array}
$$

Consider the auxiliary equation as follows:

$$
\begin{array}{r}
u(k+1)=u(k) \exp \left\{r_{1} \alpha_{1}-r_{1} u(k)\right\}, \\
k=0,1,2, \ldots
\end{array}
$$

Because of $0<r_{1} \alpha_{1} \leq 1$, according to (ii) of Lemma 5, we can obtain $u(k) \leq\left(1 / r_{1}\right)$ for all $k \geq 2$, where $u(k)$ is arbitrary positive solution of (23) with initial value $u(0)>0$. From Lemma $4, f(u)=u \exp \left(r_{1} \alpha_{1}-r_{1} u\right)$ is nondecreasing for $u \in$ $\left(0,\left(1 / r_{1}\right)\right]$. According to Lemma 6 we can obtain $x(k) \leq u(k)$ for all $k \geq 2$, where $u(k)$ is the solution of (23) with the initial value $u(2)=x(2)$. According to (i) of Lemma 5 , we can obtain

$$
U_{1}=\limsup _{k \rightarrow+\infty} x_{1}(k) \leq \lim _{k \rightarrow+\infty} u(k)=\alpha_{1} \stackrel{\text { def }}{=} M_{1}^{x_{1}} .
$$

From the second equation of system (4), we obtain

$$
\begin{array}{r}
x_{2}(k+1) \leq x_{2}(k) \exp \left\{r_{2} \alpha_{2}-r_{2} x_{2}(k)\right\}, \\
k=0,1,2, \ldots
\end{array}
$$

Similar to the above analysis, we have

$$
U_{2}=\limsup _{k \rightarrow+\infty} x_{2}(k) \leq \lim _{k \rightarrow+\infty} u(k)=\alpha_{2} \stackrel{\text { def }}{=} M_{1}^{x_{2}} .
$$

Then, for sufficiently small constant $\varepsilon>0$, there is an integer $k_{1}>2$ such that

$$
\begin{array}{r}
x_{1}(k) \leq M_{1}^{x_{1}}+\varepsilon, \quad x_{2}(k) \leq M_{1}^{x_{2}}+\varepsilon \\
\forall k>k_{1} .
\end{array}
$$

According to the first equation of system (4) we can obtain

$$
x_{1}(k+1) \geq x_{1}(k) \exp \left\{r_{1} K_{1}-r_{1} x_{1}(k)\right\} .
$$

Consider the auxiliary equation as follows:

$$
u(k+1)=u(k) \exp \left\{r_{1} K_{1}-r_{1} u(k)\right\} .
$$

Since $0<r_{1} K_{1} \leq 1$, according to (ii) of Lemma 5 , we can obtain $u(k) \leq\left(1 / K_{1}\right)$ for all $k \geq 2$, where $u(k)$ is arbitrary positive solution of (23) with initial value $u(0)>0$. From Lemma $4, f(u)=u \exp \left(r_{1} K_{1}-r_{1} u\right)$ is nondecreasing for $u \in$ $\left(0,\left(1 / K_{1}\right)\right]$. According to Lemma 6 we can obtain $x(k) \geq u(k)$ for all $k \geq 2$, where $u(k)$ is the solution of (23) with the initial value $u\left(k_{1}\right)=x\left(k_{1}\right)$. According to (i) of Lemma 5, we have

$$
V_{1}=\liminf _{k \rightarrow+\infty} x_{1}(k) \geq \lim _{k \rightarrow+\infty} u(k)=K_{1} \stackrel{\text { def }}{=} N_{1}^{x_{1}} .
$$

From the second equation of system (4), we obtain

$$
x_{2}(k+1) \geq x_{2}(k) \exp \left\{r_{2} K_{2}-r_{2} x_{2}(k)\right\} .
$$

Similar to the above analysis, we have

$$
V_{2}=\liminf _{k \rightarrow+\infty} x_{2}(k) \geq \lim _{k \rightarrow+\infty} u(k)=K_{2} \stackrel{\text { def }}{=} N_{1}^{x_{2}} .
$$

Then, for sufficiently small constant $\varepsilon>0$, there is an integer $k_{2}>k_{1}$ such that

$$
\begin{aligned}
x_{1}(k) \geq N_{1}^{x_{1}}-\varepsilon, \quad x_{2}(k) \geq & N_{1}^{x_{2}}-\varepsilon \\
& \forall k>k_{2} .
\end{aligned}
$$

Equation (24) combined with the first equation of system (4) leads to

$$
\begin{array}{r}
x_{1}(k+1) \leq x_{1}(k) \exp \left\{r_{1}\left[\frac{K_{1}+\alpha_{1}\left(M_{1}^{x_{2}}+\varepsilon\right)}{1+\left(M_{1}^{x_{2}}+\varepsilon\right)}-x_{1}(k)\right]\right\}, \\
k>k_{2} .
\end{array}
$$

Similar to the analysis of (23) and (24), we have

$$
U_{1}=\limsup _{k \rightarrow+\infty} x_{1}(k) \leq \frac{K_{1}+\alpha_{1}\left(M_{1}^{x_{2}}+\varepsilon\right)}{1+\left(M_{1}^{x_{2}}+\varepsilon\right)} .
$$

Because of arbitrariness of $\varepsilon>0$, we have $U_{1} \leq M_{2}^{x_{1}}$, where

$$
M_{2}^{x_{1}}=\frac{K_{1}+\alpha_{1} M_{1}^{x_{2}}}{1+M_{1}^{x_{2}}}<M_{1}^{x_{1}}
$$

Equation (24) combined with the second equation of system (4) leads to

$$
\begin{array}{r}
x_{2}(k+1) \leq x_{2}(k) \exp \left\{r_{2}\left[\frac{K_{2}+\alpha_{2}\left(M_{1}^{x_{1}}+\varepsilon\right)}{1+\left(M_{1}^{x_{1}}+\varepsilon\right)}-x_{2}(k)\right]\right\}, \\
k>k_{2} .
\end{array}
$$

Similar to the analysis of (23) and (24), we can obtain

$$
U_{2}=\limsup _{k \rightarrow+\infty} x_{2}(k) \leq \frac{K_{2}+\alpha_{2}\left(M_{1}^{x_{1}}+\varepsilon\right)}{1+\left(M_{1}^{x_{1}}+\varepsilon\right)} .
$$

Because of arbitrariness of $\varepsilon>0$, we have $U_{2} \leq M_{2}^{x_{2}}$, where

$$
M_{2}^{x_{2}}=\frac{K_{2}+\alpha_{2} M_{1}^{x_{1}}}{1+M_{1}^{x_{1}}}<M_{1}^{x_{2}}
$$

Then, for sufficiently small constant $\varepsilon>0$, there is an integer $k_{3}>k_{2}$ such that

$$
\begin{array}{r}
x_{1}(k) \leq M_{2}^{x_{1}}-\varepsilon, \quad x_{2}(k) \leq M_{2}^{x_{2}}-\varepsilon, \\
\forall k>k_{3} .
\end{array}
$$

Equation (27) combined with the first equation of system (4) leads to

$$
x_{1}(k+1) \geq x_{1}(k) \exp \left\{r_{1}\left[\frac{K_{1}+\alpha_{1}\left(N_{1}^{x_{2}}-\varepsilon\right)}{1+\left(N_{1}^{x_{2}}-\varepsilon\right)}-x_{1}(k)\right]\right\} \text {, }
$$

$$
k>k_{3} \text {. }
$$


From this, we can finally obtain

$$
V_{1}=\liminf _{k \rightarrow+\infty} x_{1}(k) \geq \frac{K_{1}+\alpha_{1}\left(N_{1}^{x_{2}}-\varepsilon\right)}{1+\left(N_{1}^{x_{2}}-\varepsilon\right)}
$$

Because of the arbitrariness of $\varepsilon$, we have $V_{1} \geq N_{2}^{x_{1}}$, where

$$
N_{2}^{x_{1}}=\frac{K_{1}+\alpha_{1} N_{1}^{x_{2}}}{1+N_{1}^{x_{2}}}>K_{1}=N_{1}^{x_{1}} .
$$

Equation (27) combined with the first equation of system (4) leads to

$$
\begin{array}{r}
x_{2}(k+1) \geq x_{2}(k) \exp \left\{r_{1}\left[\frac{K_{2}+\alpha_{2}\left(N_{1}^{x_{1}}-\varepsilon\right)}{1+\left(N_{1}^{x_{1}}-\varepsilon\right)}-x_{1}(k)\right]\right\}, \\
k>k_{3} .
\end{array}
$$

From the above inequality we can obtain

$$
V_{2}=\liminf _{k \rightarrow+\infty} x_{2}(k) \geq \frac{K_{2}+\alpha_{2}\left(N_{1}^{x_{1}}-\varepsilon\right)}{1+\left(N_{1}^{x_{1}}-\varepsilon\right)} .
$$

Because of the arbitrariness of $\varepsilon$, we have $V_{2} \geq N_{2}^{x_{2}}$, where

$$
N_{2}^{x_{2}}=\frac{K_{2}+\alpha_{2} N_{1}^{x_{1}}}{1+N_{1}^{x_{1}}}>K_{2}=N_{1}^{x_{2}} .
$$

Then, for sufficiently small constant $\varepsilon>0$, there is an integer $k_{4}>k_{3}$ such that

$$
\begin{array}{r}
x_{1}(k) \geq N_{2}^{x_{1}}-\varepsilon, \quad x_{2}(k) \geq N_{2}^{x_{2}}-\varepsilon \\
\forall k>k_{4} .
\end{array}
$$

Continuing the above steps, we can get four sequences $\left\{M_{k}^{x_{1}}\right\},\left\{M_{k}^{x_{2}}\right\},\left\{N_{k}^{x_{1}}\right\}$, and $\left\{N_{k}^{x_{1}}\right\}$ such that

$$
\begin{array}{rlrl}
M_{k}^{x_{1}} & =\frac{K_{1}+\alpha_{1} M_{k-1}^{x_{2}}}{1+M_{k-1}^{x_{2}}}, & M_{k}^{x_{2}}=\frac{K_{2}+\alpha_{2} M_{k-1}^{x_{1}}}{1+M_{k-1}^{x_{1}}} ; \\
N_{k}^{x_{1}}=\frac{K_{1}+\alpha_{1} N_{k-1}^{x_{2}}}{1+N_{k-1}^{x_{2}}}, & N_{k}^{x_{2}}=\frac{K_{2}+\alpha_{2} N_{k-1}^{x_{1}}}{1+N_{k-1}^{x_{1}}} .
\end{array}
$$

Clearly, we have

$$
\begin{gathered}
N_{k}^{x_{1}} \leq V_{1} \leq U_{1} \leq M_{k}^{x_{1}}, \\
N_{k}^{x_{2}} \leq V_{2} \leq U_{2} \leq M_{k}^{x_{2}}, \\
k=0,1,2, \ldots
\end{gathered}
$$

Now, we will prove $\left\{M_{k}^{x_{i}}\right\}(i=1,2)$ is monotonically decreasing and $\left\{N_{k}^{x_{i}}\right\}(i=1,2)$ is monotonically increasing by means of inductive method.
First of all, it is clear that $M_{2}^{x_{i}} \leq M_{1}^{x_{i}}, N_{2}^{x_{i}} \geq N_{1}^{x_{i}}(i=1,2)$. For $i \geq 2$, we assume that $M_{i}^{x_{1}} \leq M_{i-1}^{x_{1}}$ and $N_{i}^{x_{1}} \geq N_{i-1}^{x_{1}}$ hold; then

$$
\begin{gathered}
M_{i+1}^{x_{2}}=\frac{K_{2}+\alpha_{2} M_{i}^{x_{1}}}{1+M_{i}^{x_{1}}} \leq \frac{K_{2}+\alpha_{2} M_{i-1}^{x_{1}}}{1+M_{i-1}^{x_{1}}}=M_{i}^{x_{2}}, \\
N_{i+1}^{x_{2}}=\frac{K_{2}+\alpha_{2} N_{i}^{x_{1}}}{1+N_{i}^{x_{1}}} \geq \frac{K_{2}+\alpha_{2} N_{i-1}^{x_{1}}}{1+N_{i-1}^{x_{1}}}=N_{i}^{x_{2}}, \\
M_{i+1}^{x_{1}}=\frac{K_{1}+\alpha_{1} M_{i}^{x_{2}}}{1+M_{i}^{x_{2}}} \leq \frac{K_{1}+\alpha_{1} M_{i-1}^{x_{2}}}{1+M_{i-1}^{x_{2}}}=M_{i}^{x_{1}}, \\
N_{i+1}^{x_{1}}=\frac{K_{1}+\alpha_{1} N_{i}^{x_{2}}}{1+N_{i}^{x_{2}}} \geq \frac{K_{1}+\alpha_{1} N_{i-1}^{x_{2}}}{1+N_{i-1}^{x_{1}}}=N_{i}^{x_{1}} .
\end{gathered}
$$

Equations (51)-(54) show that $\left\{M_{k}^{x_{1}}\right\}$ and $\left\{M_{k}^{x_{2}}\right\}$ are monotonically decreasing and $\left\{N_{k}^{x_{1}}\right\}$ and $\left\{N_{k}^{x_{2}}\right\}$ are monotonically increasing. Consequently, $\lim _{k \rightarrow+\infty}\left\{M_{k}^{x_{i}}\right\}$ and $\lim _{k \rightarrow+\infty}\left\{N_{k}^{x_{i}}\right\}(i=1,2)$ both exist. Let

$$
\lim _{k \rightarrow+\infty} M_{k}^{x_{i}}=X_{i}^{*}, \quad \lim _{k \rightarrow+\infty} N_{k}^{x_{i}}=x_{i}^{*}, \quad i=1,2 .
$$

From (48) and (55), we have

$$
\begin{aligned}
& X_{1}^{*}=\frac{K_{1}+\alpha_{1} X_{2}^{*}}{1+X_{2}^{*}}, \\
& X_{2}^{*}=\frac{K_{2}+\alpha_{2} X_{1}^{*}}{1+X_{1}^{*}} .
\end{aligned}
$$

From (49) and (55), we get

$$
\begin{aligned}
& x_{1}^{*}=\frac{K_{1}+\alpha_{1} x_{2}^{*}}{1+x_{2}^{*}}, \\
& x_{2}^{*}=\frac{K_{2}+\alpha_{2} x_{1}^{*}}{1+x_{1}^{*}} .
\end{aligned}
$$

Equations (56) and (57) show that $\left(X_{1}^{*}, X_{2}^{*}\right)$ and $\left(x_{1}^{*}, x_{2}^{*}\right)$ are all solutions of system (6). However, system (6) has unique positive solution $\left(\bar{x}_{1}, \bar{x}_{2}\right)$. Therefore

$$
U_{i}=V_{i}=\lim _{k \rightarrow+\infty} x_{i}(k)=\bar{x}_{i}, \quad i=1,2
$$

That is, $E_{+}\left(\bar{x}_{1}, \bar{x}_{2}\right)$ is globally attractive.

From Theorem 3 , we get that equilibrium $E_{+}\left(\bar{x}_{1}, \bar{x}_{2}\right)$ is locally asymptotically stable. And so, $E_{+}\left(\bar{x}_{1}, \bar{x}_{2}\right)$ is globally asymptotically stable. This ends the proof of Theorem 1. 


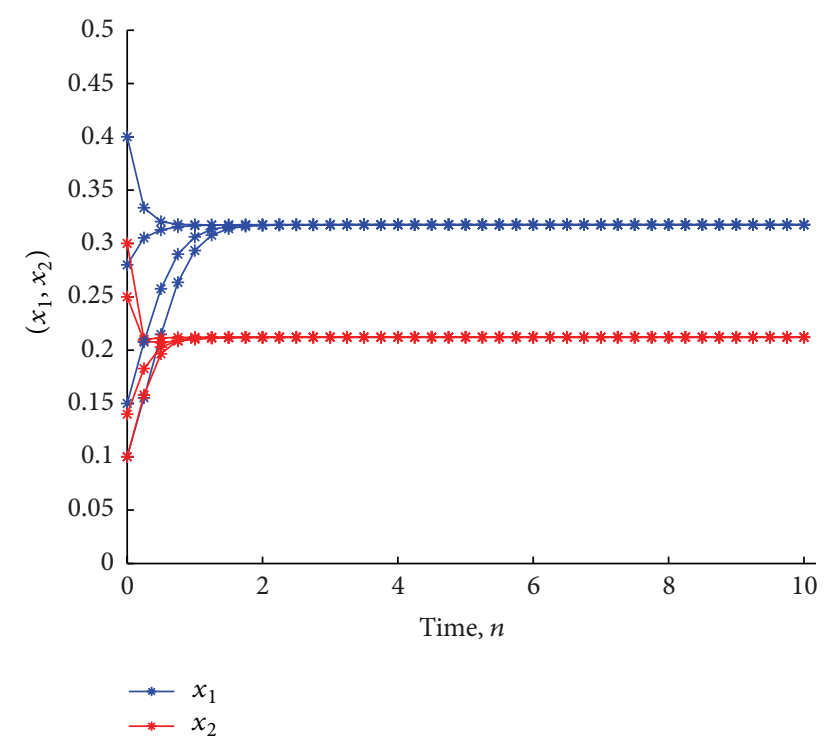

FIgURE 1: Dynamic behaviors of the solution $\left(x_{1}(t), x_{2}(t)\right)$ of system (59), with the initial conditions $\left(x_{1}(0), x_{2}(0)\right)=(0.1,0.25)$, $(0.28,0.3),(0.15,0.14)$, and $(0.4,0.1)$, respectively.

\section{Example}

In this section, we will give an example to illustrate the feasibility of the main result.

Example. Consider the following cooperative system:

$$
\begin{aligned}
& x_{1}(k+1)=x_{1}(k) \exp \left\{2\left[\frac{0.3+0.4 x_{2}(k)}{1+x_{2}(k)}-x_{1}(k)\right]\right\}, \\
& x_{2}(k+1)=x_{2}(k) \exp \left\{4\left[\frac{0.2+0.25 x_{1}(k)}{1+x_{1}(k)}-x_{2}(k)\right]\right\} .
\end{aligned}
$$

By calculating, we have that positive equilibrium $E_{+}\left(\bar{x}_{1}, \bar{x}_{2}\right)=$ (0.317495, 0.212049), $r_{1} \alpha_{1}=0.8<1, r_{2} \alpha_{2}=1, K_{i}<\alpha_{i}(i=$ $1,2)$ and the coefficients of system (59) satisfy $\left(H_{1}\right)$ and $\left(H_{2}\right)$. From Theorem 1, positive equilibrium $E_{+}\left(\bar{x}_{1}, \bar{x}_{2}\right)$ is globally asymptotically stable. Numeric simulation also supports our finding (see Figure 1).

\section{Discussion}

It is well known [6] that, for autonomous two-species LotkaVolterra mutualism model, the conditions which ensure the existence of positive equilibrium are enough to ensure that the equilibrium is globally stable. However, for the twospecies discrete Lotka-Volterra mutualism model, Lu and Wang [22] proved that a cooperative system cannot be permanent. That is, the dynamic behaviors of discrete LotkaVolterra mutualism model are very different to the continuous ones.

Recently, by using the iterative method, Xie et al. [8] showed that, for a mutualism model with infinite delay, conditions which ensure the permanence of the system are enough to ensure the global stability of the system. As a corollary of their result, one could draw the conclusion that system (1) admits a unique positive equilibrium, which is globally stable. One interesting issue is proposed. For the discrete type mutualism model (4), is there any relationship between the existence of positive equilibrium and the stability property of the positive equilibrium?

In this paper, by using the linear approximation, comparison principle of difference equations, and method of iteration scheme, we showed that the conditions which ensure the local stability property of the positive equilibrium $\left(\left(\mathrm{H}_{2}\right) 0<r_{i} \alpha_{i} \leq\right.$ $1(i=1,2))$ are also enough to guarantee the global stability of the positive equilibrium $E_{+}\left(\bar{x}_{1}, \bar{x}_{2}\right)$.

At the end of this paper, we would like to mention here that, for the Lotka-Volterra type mutualism system with time delay, delay is one of the most important factors to influence the dynamic behaviors of the system [23-25]. It seems interesting to incorporate the time delay to the system (4) and investigate the dynamic behaviors of the system; we leave this for future study.

\section{Conflict of Interests}

The authors declare that there is no conflict of interests regarding the publication of this paper.

\section{Acknowledgments}

The research was supported by the Natural Science Foundation of Fujian Province (2013J01011, 2013J01010) and the Foundation of Fujian Education Bureau (JA13361).

\section{References}

[1] L. J. Chen, L. J. Chen, and Z. Li, "Permanence of a delayed discrete mutualism model with feedback controls," Mathematical and Computer Modelling, vol. 50, no. 7-8, pp. 1083-1089, 2009.

[2] Y. Li, "On a periodic mutualism model," The ANZIAM Journal, vol. 42, no. 4, pp. 569-580, 2001.

[3] Y. K. Li and G. T. Xu, "Positive periodic solutions for an integrodifferential model of mutualism," Applied Mathematics Letters, vol. 14, no. 5, pp. 525-530, 2001.

[4] F. Chen and M. You, "Permanence for an integrodifferential model of mutualism," Applied Mathematics and Computation, vol. 186, no. 1, pp. 30-34, 2007.

[5] F. Chen, J. Yang, L. Chen, and X. Xie, "On a mutualism model with feedback controls," Applied Mathematics and Computation, vol. 214, no. 2, pp. 581-587, 2009.

[6] F. D. Chen and X. X. Xie, Study on the Dynamic Behaviors of Cooperative System, Science Press, Beijing, China, 2014.

[7] F. Y. Wei and C. Y. Li, "Permanence and globally asymptotic stability of cooperative system incorporating harvesting," Advances in Pure Mathematics, vol. 3, no. 7, pp. 627-632, 2013.

[8] X. D. Xie, F. D. Chen, K. Yang, and Y. Xue, "Global attractivity of an integrodifferential model of mutualism," Abstract and Applied Analysis, vol. 2014, Article ID 928726, 6 pages, 2014.

[9] X. D. Xie, F. D. Chen, and Y. L. Xue, "Note on the stability property of a cooperative system incorporating harvesting," Discrete Dynamics in Nature and Society, vol. 2014, Article ID 327823, 5 pages, 2014. 
[10] Y. K. Li, "Positive periodic solutions of a discrete mutualism model with time delays," International Journal of Mathematics and Mathematical Sciences, no. 4, pp. 499-506, 2005.

[11] F. D. Chen, "Permanence for the discrete mutualism model with time delays," Mathematical and Computer Modelling, vol. 47, no. 3-4, pp. 431-435, 2008.

[12] A. Muhammadhaji and Z. Teng, "Global attractivity of a periodic delayed $N$-species model of facultative mutualism," Discrete Dynamics in Nature and Society, vol. 2013, Article ID 580185, 11 pages, 2013.

[13] A. Muhammadhaji and T. Niyaz, "Positive periodic solutions of cooperative systems with delays and feedback controls," International Journal of Differential Equations, vol. 2013, Article ID 502963, 9 pages, 2013.

[14] G. C. Lu, Z. Y. Lu, and X. Lian, "Delay effect on the permanence for Lotka-Volterra cooperative systems," Nonlinear Analysis: Real World Applications, vol. 11, no. 4, pp. 2810-2816, 2010.

[15] Z. J. Liu, R. H. Tan, Y. P. Chen, and L. S. Chen, “On the stable periodic solutions of a delayed two-species model of facultative mutualism," Applied Mathematics and Computation, vol. 196, no. 1, pp. 105-117, 2008.

[16] W. Yang and X. Li, "Permanence of a discrete nonlinear $N$ species cooperation system with time delays and feedback controls," Applied Mathematics and Computation, vol. 218, no. 7, pp. 3581-3586, 2011.

[17] X. Li and W. Yang, "Permanence of a discrete model of mutualism with infinite deviating arguments," Discrete Dynamics in Nature and Society, vol. 2010, Article ID 931798, 7 pages, 2010.

[18] Y. Liao and T. Zhang, "Almost periodic solutions of a discrete mutualism model with variable delays," Discrete Dynamics in Nature and Society, vol. 2012, Article ID 742102, 27 pages, 2012.

[19] C. Xu and Y. Wu, "Permanence in a discrete mutualism model with infinite deviating arguments and feedback controls," Discrete Dynamics in Nature and Society, vol. 2013, Article ID 397382, 7 pages, 2013.

[20] G. Chen and Z. Teng, "On the stability in a discrete twospecies competition system," Journal of Applied Mathematics and Computing, vol. 38, no. 1-2, pp. 25-39, 2012.

[21] L. Wang and M. Q. Wang, Ordinary Difference Equation, Xinjing University Press, Urmuqi, China, 1989.

[22] Z. Lu and W. Wang, "Permanence and global attractivity for Lotka-Volterra difference systems," Journal of Mathematical Biology, vol. 39, no. 3, pp. 269-282, 1999.

[23] X. He and K. Gopalsamy, "Persistence, attractivity, and delay in facultative mutualism," Journal of Mathematical Analysis and Applications, vol. 215, no. 1, pp. 154-173, 1997.

[24] D. Mukherjee, "Permanence and global attractivity for facultative mutualism system with delay," Mathematical Methods in the Applied Sciences, vol. 26, no. 1, pp. 1-9, 2003.

[25] Y. Nakata and Y. Muroya, "Permanence for nonautonomous Lotka-Volterra cooperative systems with delays," Nonlinear Analysis. Real World Applications, vol. 11, no. 1, pp. 528-534, 2010 . 


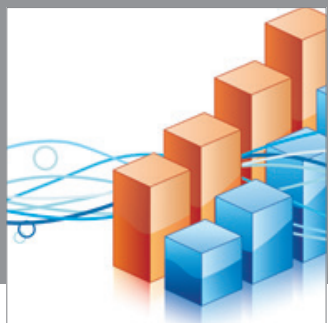

Advances in

Operations Research

mansans

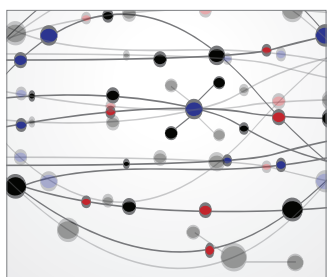

The Scientific World Journal
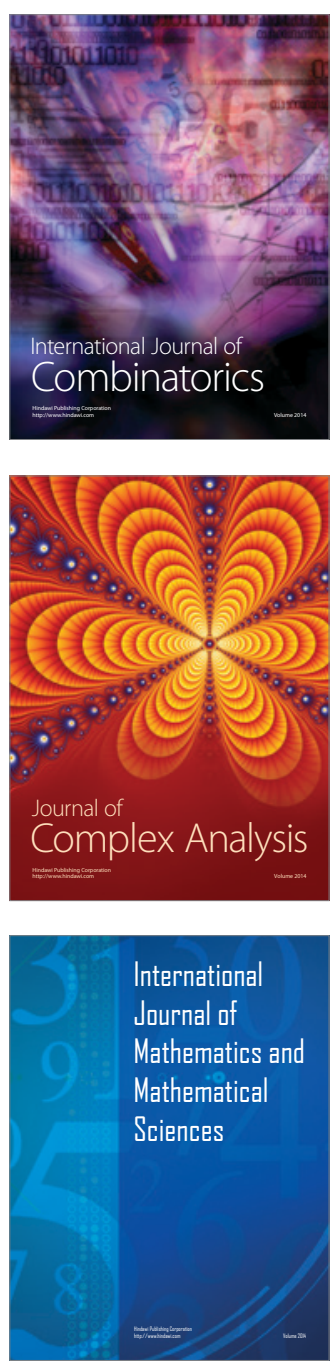
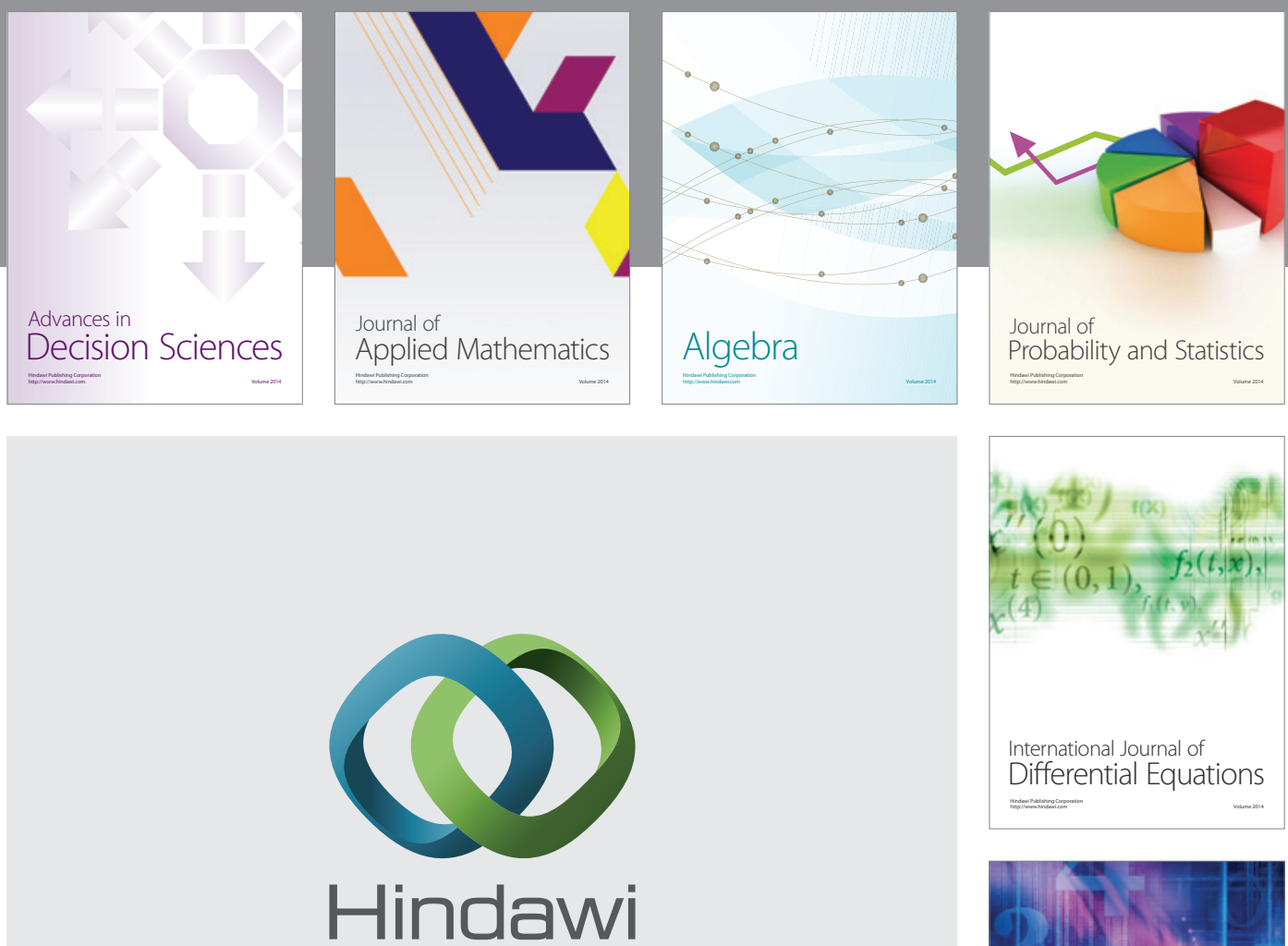

Submit your manuscripts at http://www.hindawi.com
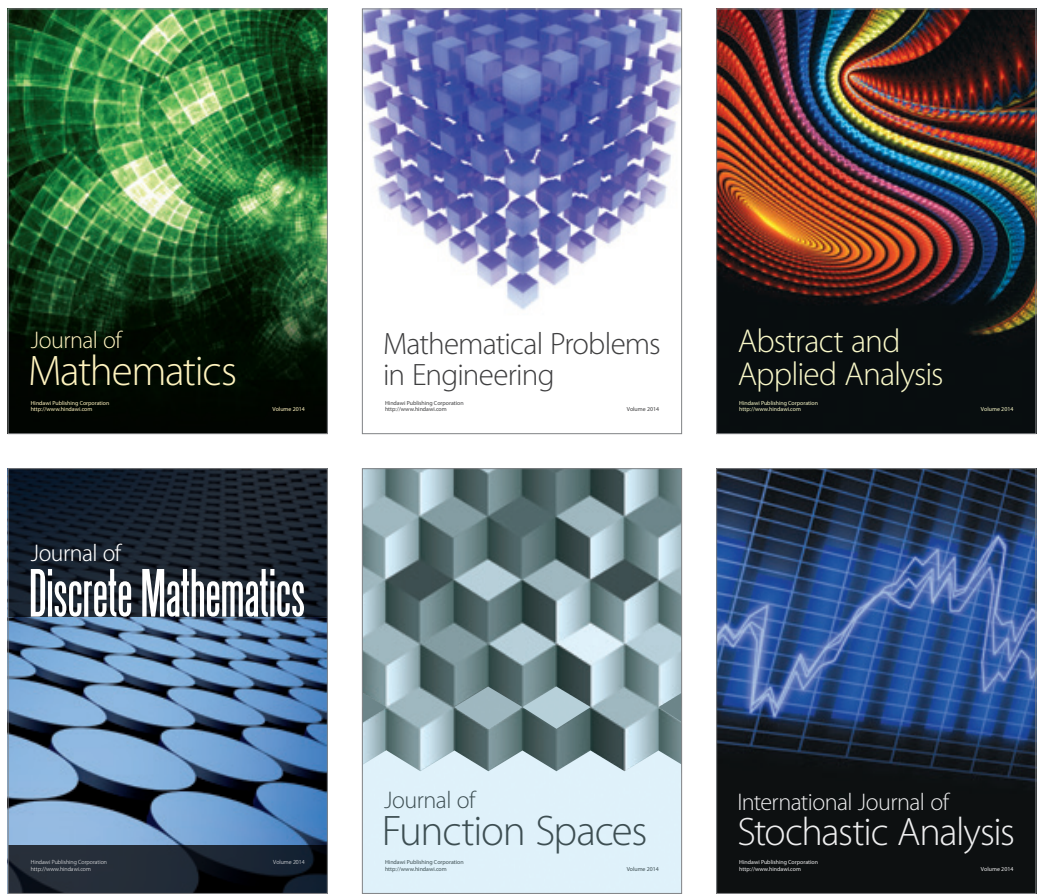

Journal of

Function Spaces

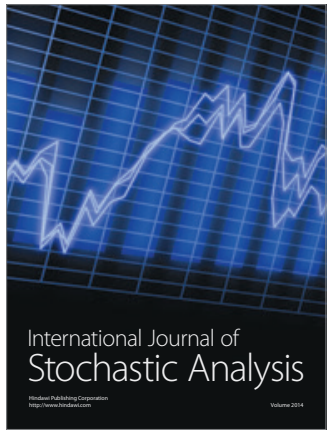

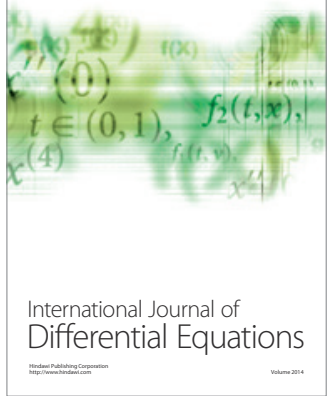
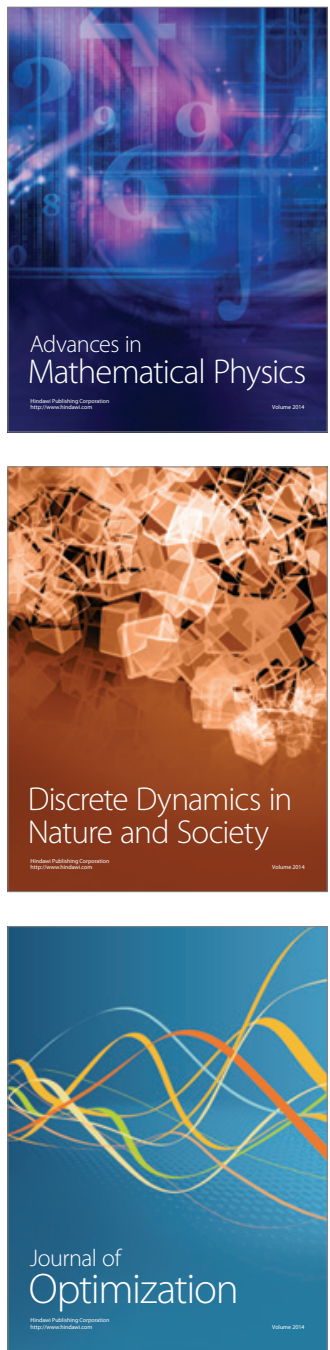